Parker, M. T. \& Simmons, L. E. (1959). J. gen. Microbiol. 21, 457-476

\title{
The Inhibition of Corynebacterium diphtheriae and other Gram-positive Organisms by Staphylococcus aureus
}

\author{
BY M. T. PARKER AND L. E. SIMMONS \\ Public Health Laboratory, Manchester, 10
}

\begin{abstract}
SUMMARY: A large collection of Staphylococcus aureus strains of human origin was examined for ability to inhibit the growth of Corynebacterium diphtheriae on the surface of solid media. Inhibition with the formation of a sharply-defined zone was produced by members of $S$. aureus Type 71, by certain closely-related strains, and by very few others. A small number of strains gave a wider hazy zone of inhibition. Both types of zone were produced by agents which diffused through agar; but not through cellophan, and killed C. diphtheriae. The two sorts of agent differed in their spectrum of activity for other corynebacteria. Strains of $S$. aureus which produced sharp zones of inhibition of $C$. diphtheriae also had a weak inhibitory activity against strains of $S$. aureus which were non-inhibitors or producers of hazy zones, but not on others which produced sharp zones. Ability to give rise to hazy zones persisted indefinitely in culture, but ability to produce sharp zones was regularly lost in culture after a few months. Loss of activity was accompanied by a widening of the susceptibility of the staphylococci to lysis by typing phages.
\end{abstract}

It has been known for many years that some staphylococci are able to inhibit the growth of Corynebacterium diphtheriae. Dujardin-Beaumetz (1932) showed that staphylococci which possessed this property were also active against a number of other Gram-positive cocci, and that their inhibitory effect was only demonstrable on solid media. Duliscouët $(1935,1939,1945)$ confirmed the inhibitory action of some staphylococci on corynebacteria and in addition described a stimulating effect produced by others. He showed that inhibition was due to the production during growth of a substance which diffused out into the medium. Both of these workers used a technique of 'simultaneous antagonism' (Gratia, 1946) in which spots or streaks of staphylococci were deposited on a solid medium immediately after its surface had been evenly sown with an inoculum of corynebacteria. Several later workers investigated the inhibitory activities of Gram-positive organisms by using more sensitive methods similar to those which Gratia \& Fredericq used for the study of colicines (see Fredericq, 1948). In these 'deferred antagonism' techniques (Gratia, 1946), the strain being tested for inhibitory activity was grown on a solid medium for one or more days before the strain to be inhibited was inoculated. In this way a much greater number of active strains were detected, and it became apparent that a complex pattern of antagonisms existed not only between staphylococci and corynebacteria (Jennings \& Sharp, 1947) but also between staphylococci and other staphylococci (Fredericq, 1946; Halbert, Swick \& Sonn, 1953) and between corynebacteria and corynebacteria (Thibaut 1949; Terrasse \& Sohier, 1954). The 
possession of inhibitory activity appeared, however, to be distributed in a haphazard fashion among members of each of these bacterial groups, and the production of a particular inhibitory substance was in no instance characteristic of a particular species or type of organism.

Parker, Tomlinson \& Williams (1955) found that Staphylococcus aureus strains belonging to a well-defined type, which could be identified by susceptibility to a single phage (Type 71), were closely associated with impetigo contagiosa, and to a lesser extent with superficial vesicular skin infections of newborn infants, but were rarely seen in deep lesions. Many years before, A. J. H. Tomlinson (unpublished investigation) had observed that over half of the strains of $S$. aureus from impetigo lesions, and a much smaller proportion from other sources, inhibited the growth of corynebacteria. He used a simultaneous antagonism technique in which loopfuls of staphylococcal broth cultures were superimposed on a freshly-inoculated lawn of a diphtheroid on a blood agar plate: after overnight incubation, the areas of staphylococcal growth were surrounded by zones of inhibition of the corynebacterium. When $S$. aureus Type 71 strains were tested in this way, it was found that $90 \%$ of them inhibited the growth of a strain of Corynebacterium diphtheriae; most other strains of $S$. aureus showed no inhibitory action. It was also noticed that staphylococci could give rise to one of two quite different sorts of inhibitory zone on lawns of $C$. diphtheriae. The zones formed by $S$. aureus Type 71 strains were always sharply defined and rather narrow (2-4 $\mathrm{mm}$. radius), but those formed by a number of other strains were wider (more than $5 \mathrm{~mm}$. radius) and hazy at the periphery. Later investigations (Parker, 1958) showed that most of the few strains, other than members of Type $\pi 1$, which produced narrow sharp zones had phage patterns not very different from that of Type 71, had a number of other ' 71 -like' characters, and were also associated with impetigo and other superficial vesicular skin infections.

The present communication summarizes observations made in the course of this work on the properties of these inhibitory agents, and on the associated changes which take place in a strain of Staphylococcus aureus when the ability to inhibit Corynebacterium diphtheriae is lost.

\section{METHODS}

\section{Organisms}

Coagulase-positive staphylococci. Strains (2032) of coagulase-positive staphylococci isolated in the laboratory or received from elsewhere during 1953-57 were tested for ability to inhibit the growth of Corynebacterium diphtheriae, and were phage typed by the method described by Anderson \& Williams (1956). All strains lysed by phage 71 either at the routine test dilution (RTD) or at $\times 1000$ the strength of the routine test dilution $(1000 \times$ RTD), but not lysed by any other phage at the RTD, were included in Type 71.

Coagulase-negative staphylococci. Twenty-two strains of coagulase-negative, catalase-positive, Gram-positive cocci isolated from nasal swabs or from skin lesions were used in various tests for susceptibility to the action of Staphylococcus aureus cultures. 
Corynebacteria. The strain used in the routine inhibition test was a nitratenegative, non-toxigenic strain of Corynebacterium diphtheriae mitis, No. 51 in the collection kept in this Laboratory. Thirty-nine other corynebacteria, listed in Section B2, p. 466 were used in inhibition tests with a limited number of strains of Staphylococcus aureus.

Other organisms. Twelve strains of haemolytic streptococci (4 each of Groups A, C and G), and 2 strains of pneumococci isolated from routine material, were used in various inhibition tests.

\section{Methods used in the study of inhibition on solid media}

(a) Simultaneous antagonism (Gratia, 1925, 1946). In these methods, the active strain was deposited on the surface of an agar medium over which the sensitive (or passive) strain had been recently spread. Such a procedure was only of value when the inhibitory substance was powerful and was produced promptly.

(i) The routine inhibition test. Before use, the test strain of Corynebacterium diphtheriae had been subcultured several times at daily intervals on blood agar. In earlier experiments the surface of a blood agar plate was sown with $C$. diphtheriae by spreading a loopful of growth from a previous blood agar plate as evenly as possible over the whole surface, but later it was found that a more even sheet of growth of the correct thickness could be obtained by flooding the plate with a broth emulsion of growth from blood agar, roughly standardized to the opacity of Brown's No. 3 tube. As soon as the surface of the plate was dry, small loopfuls of overnight broth cultures of staphylococci were placed in rows on it. After overnight incubation at $37^{\circ}$ the corynebacterium had grown as an even confluent sheet, with zones of inhibition around some of the circular areas of staphylococcal growth. The radius and other characters of the zones were recorded. The inhibition test was considered positive $(\mathrm{DI}+$ ) when the zone was $1 \mathrm{~mm}$. or more in radius. The effect of variations in the technique of the test will be discussed on pp. 462-4.

(ii) The overlapping drop technique. This method was based on the crosstitration procedure of Rosebury, Gale \& Taylor (1954) in which pairs of overlapping drops of broth cultures were placed on a solid medium. Drops were deposited with a standard loop on a blood agar plate: the second drop of each pair was added as soon as the first had dried and overlapped the first by approximately $\frac{1}{3}$ of its area. Plates were examined for inhibition extending beyond the area of overlap after $18 \mathrm{hr}$. at $37^{\circ}$. This was a useful method for examining each of a group of cultures for inhibition of, or by, every other member of the group.

(b) Deferred antagonism (Gratia, 1946). In these methods the active strain was allowed to grow on a solid medium for 1 or more days before the sensitive strain was inoculated. They were designed to detect inhibitory substances weaker than those revealed by techniques of simultaneous antagonism.

(i) Direct streaking methods. The active strain was grown as a spot at the centre of a plate. After incubation, passive strains were streaked up to the 
primary growth as a series of radial lines. It proved difficult to inoculate the passive strains close enough to the primary spot to detect weak inhibition, but the method was useful for measuring the rate of diffusion of inhibitory agents through agar.

(ii) Methods employing chloroform. Gratia (1925) found colicines to be resistant to prolonged contact with chloroform, and Fredericq (1948) grew active strains as central spots, killed them with chloroform, and inoculated the whole surface with a passive strain. We used this method in earlier experiments but later adopted a more convenient variation which had been developed in this Laboratory for the colicine typing of Escherichia coli 055 and Shigella sonnei (Shannon, 1957; Abbott \& Shannon, 1958). A streak of growth of the active strain was inoculated across the centre of a blood agar plate and incubated at $37^{\circ}$ for $48 \mathrm{hr}$. The Petri dish was then placed lid downwards on the bench and a circular piece of filter paper inserted and saturated with chloroform. After $1 \mathrm{hr}$. the growth was scraped to one end of the plate with the edge of a clean glass slide and removed, together with a small piece of agar. More chloroform was then added to the paper in the lid and the plate left over it for a further $1 \mathrm{hr}$. Then the paper was removed and the plate exposed to the air face downwards for $2 \mathrm{hr}$. Loopfuls of broth cultures of passive strains were then streaked across the medium at right angles to the position formerly occupied by the active strain. After overnight incubation at $37^{\circ}$ the plates were examined for evidence of inhibition of the passive strains near the centre of the lines of growth.

(iii) Cellophan method. Autoclaved sheets of commercial cellophan were placed on the surface of blood agar plates and allowed to dry. A spot of staphylococcal broth culture was placed in the centre of each cellophan sheet and the plates were incubated for various periods at different temperatures. The cellophan sheets were then removed, the surface of the medium was flooded with a broth suspension of Corynebacterium diphtheriae, and the plates incubated overnight at $37^{\circ}$.

(iv) Filter-paper method (Gratia, 1946). When it was desired to detect inhibitory substances not able to pass through cellophan, a sheet of filter paper was placed on the surface of a blood agar plate and a layer of molten nutrient agar poured on top. A drop of staphylococcal broth culture was placed in the centre of the plate, which was then incubated. The top layer of agar was then stripped off with the filter-paper, so removing all the bacteria, and the underlying blood agar was flooded with a suspension of Corynebacterium diphtheriae.

\section{RESULTS}

Inhibition of Corynebacterium diphtheriae No. 51 by Staphylococcus aureus Distribution of inhibitory properties among Staphylococcus aureus strains from various sources. The routine inhibition test was used to study the distribution of inhibitory properties among a large collection of Staphylococcus aureus strains of human origin. It was not a highly sensitive method, but it gave very clear cut results and the distinction between the sharp and the hazy type 

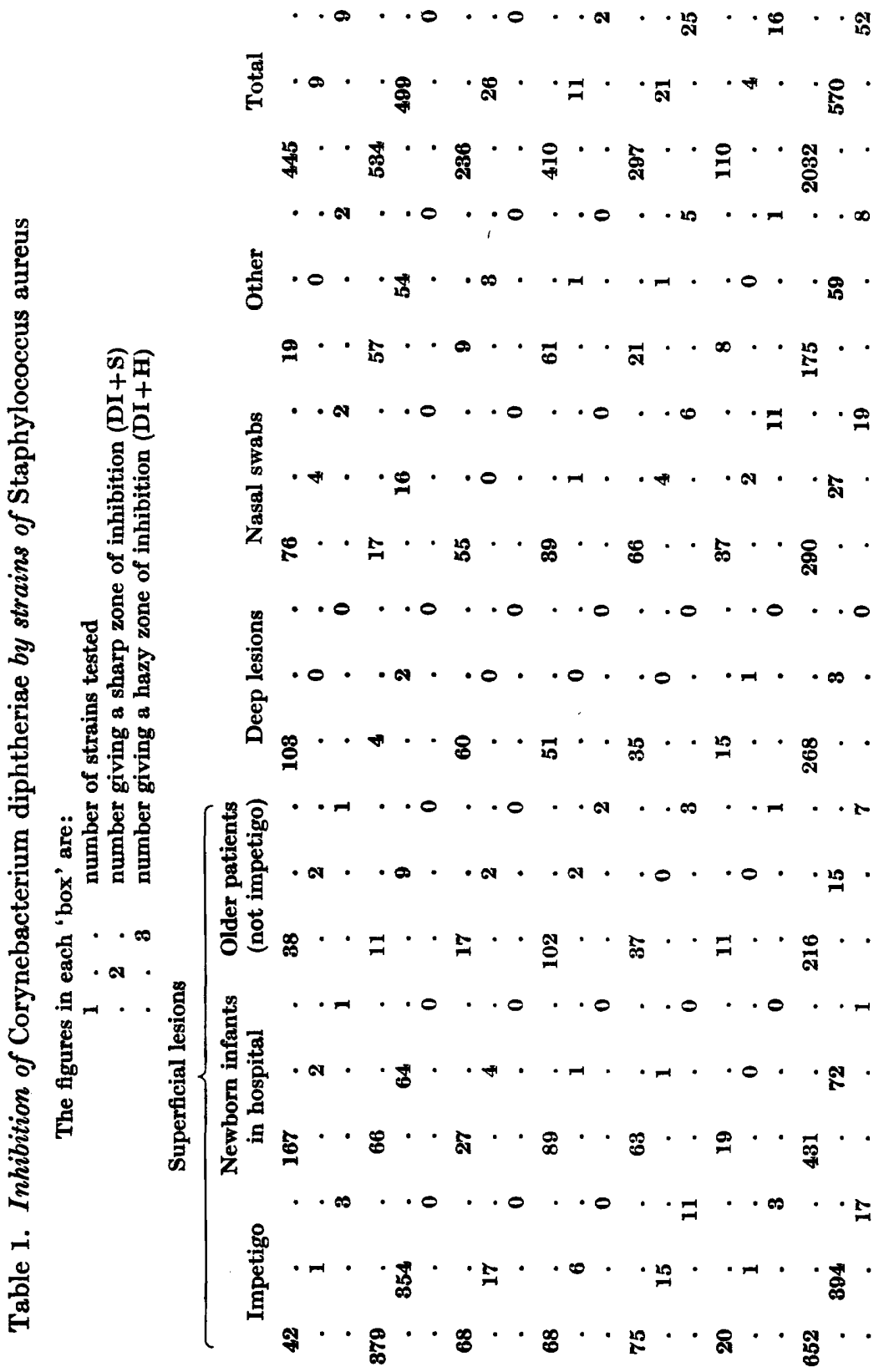

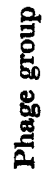

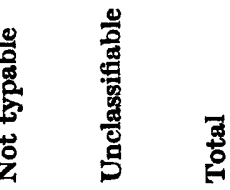


of zone was easily seen ( $\mathrm{Pl}$. 1). Table 1 summarizes the results of tests on 2032 strains, showing the numbers giving sharp zones $(D I+S)$ and the number giving hazy zones $(\mathrm{DI}+\mathrm{H})$ in the various phage groups, and from different sources. The main points of interest are displayed in Figs. 1 and 2.

Over $93 \%$ of all Staphylococcus aureus Type 71 strains were $\mathrm{DI}+$, and all positive reactors formed sharp zones. Most of the small number of other DI + S strains were either members of Group II other than Type 71 or were non-typable, and most had been isolated from impetigo or from vesicular skin lesions of new-born infants. As described elsewhere (Parker, 1958), these strains had other ' 71 -like' cultural characters, and were in the main egg-yolk negative, producers of opacity on serum agar plates and penicillin resistant.
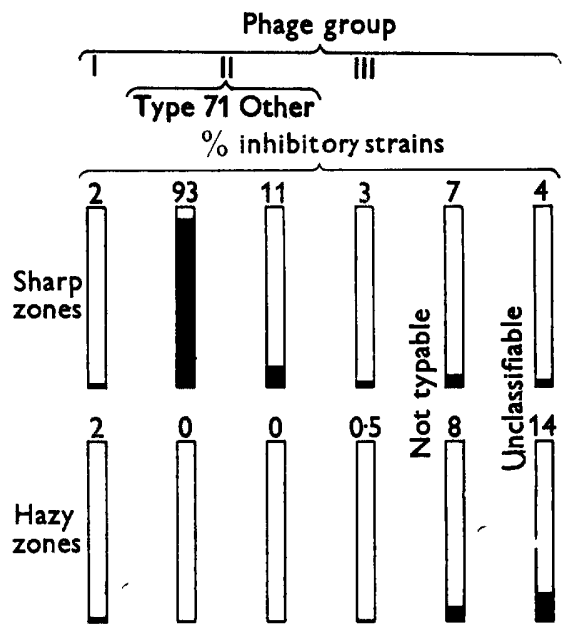

Fig. 1

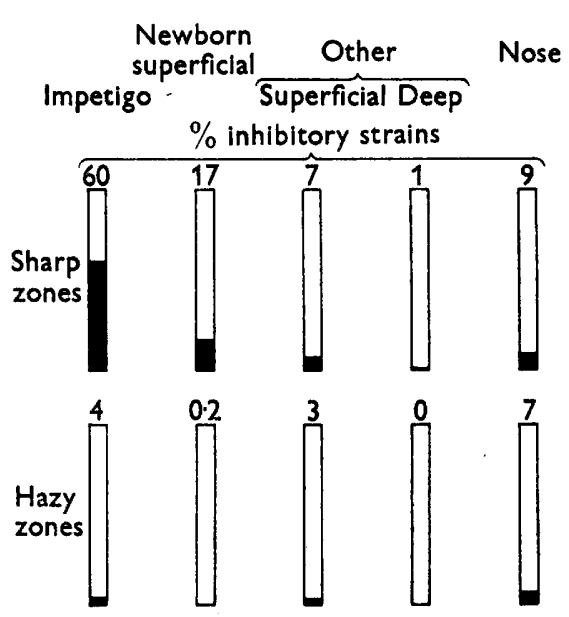

Fig. 2

Fig. 1. Inhibition of Corynebacterium diphtheriae by Staphylococcus aureus. Percentage of inhibitory strains in phage groups is shown.

Fig. 2. Inhibition of Corynebacterium diphtheriae by Staphylococcus aureus. Percentage of inhibitory strains from different sources.

Only $2 \cdot 6 \%$ of all strains formed hazy zones. Some $\mathrm{DI}+\mathrm{H}$ strains occurred in all phage groups except II, but the majority were either unclassifiable or non-typable; those which could be typed showed a wide variety of patterns. They were found most commonly in nasal swabs from normal people and in superficial lesions in which they appeared to be secondary invaders. Some were penicillin sensitive and some resistant and most, but not all, were eggyolk positive.

A few tests were made with the more sensitive Gratia-Fredericq chloroform method, with incubation of the staphylococcal cultures for $48 \mathrm{hr}$. before Corynebacterium diphtheriae was added. These tests revealed the presence of numerous weaker inhibitory agents produced by strains other than those positive in the routine test. Their distribution did not appear to have any relation to the other characteristics under investigation, and these strains were not investigated further. 


\section{Nature of the inhibitory action of Staphylococcus aureus on Corynebacterium diptheriae}

The inhibitory action of Staphylccoccus aureus on Corynebacterium diphtheriae was studied in experiments in which they were grown together on solid medium. The fact that the zone of inhibition extended for several $\mathrm{mm}$. beyond the staphylococcal macrocolony suggested that inhibition was due to diffusion of some substance formed by the active staphylococcal strain during growth. Inhibitory activity was also demonstrated with $\mathrm{DI}+\mathrm{S}$ and $\mathrm{DI}+\mathrm{H}$ strains, in absence of living staphylococci, either on plates which had been treated with chloroform or in the depths of an agar sheet on the surface of which staphylococci had been grown and later removed by Gratia's filterpaper method. In both types of experiment the inhibition of $C$. diphtheriae extended $0 \cdot 5-1 \mathrm{~cm}$. beyond the area originally occupied by the staphylococcus. The rate of diffusion of inhibitory agents was studied by spot-inoculating a staphylococcus at the centre of a plate, and streaking a loopful of a broth suspension of $C$. diphtheriae up to it on each of several successive days. There appeared to be no gross difference in the rate of diffusion of the inhibitory agents of $\mathrm{DI}+\mathrm{S}$ and $\mathrm{DI}+\mathrm{H}$ staphylococcal strains after the first day; zones of inhibition up to $2.5 \mathrm{~cm}$. in width were seen when the corynebacterium was inoculated after 5 days' incubation of the plate. None of the inhibitory agents tested was able to diffuse through cellophan.

Blood agar was chosen as the medium for the routine test, but inhibition occurred also on a number of other media. Good zones were formed on serum agar, but the distinction between sharp and hazy zones was not easily seen. Corynebacterium diphtheriae No. 51 grew poorly on plain agar, but with diphtheroids which were able to grow well in the absence of blood there was little difference in the size of the zones formed on blood agar or on plain agar. Inhibition occurred also on plates incubated anaerobically.

The outer edge of the sharp zone seen in the routine inhibition test was formed by an almost vertical wall of growth of the corynebacterium; the floor of the inhibition zone appeared at first sight to be devoid of bacterial growth but when it was touched lightly with a wire it was seen to be covered with a fine film. The inner $2-3 \mathrm{~mm}$. of a hazy zone was similarly covered with a fine film and beyond this the growth became progressively thicker as it merged into the surrounding lawn. Microscopic examination of the fine film of growth showed the presence of numerous bacilli. Although Corynebacterium diphtheriae No. 51 normally had a typical mitis morphology on blood agar, these organisms in the fine film were shorter, more pointed, stained very poorly, and were beaded. Subculture from the fine film was invariably sterile. In the sharp inhibition zones no growth was obtained until the outer, perpendicular wall of the zone was touched: in the hazy zones, subcultures from the inner $\mathbf{3} \mathbf{~ m m}$. were also sterile, but small numbers of organisms could be grown from the intermediate areas of partial inhibition. On continued incubation of plates beyond the first day there was no further extension of the zones, and no colonies of resistant organisms appeared in them. 
In the routine inhibition test plates were flooded with a thick suspension of Corynebacterium diphtheriae. This gave rise to an even confluent sheet of growth in which the distinctive characters of the sharp and the hazy type of zone could be well seen. Further increases in the concentration of the suspension had little effect on the final result, but decreases in concentration resulted in a progressive increase in zone size.

With DI +S staphylococcal strains, each 100-fold decrease in concentration of the corynebacterium increased the zone radius by about $1 \mathrm{~mm}$., up to a maximum of 6-7 mm., and the character of the zone was unaltered; but with $\mathrm{DI}+\mathrm{H}$ staphylococcal strains the effect of decreasing the size of inoculum of C. diphtheriae was to alter the character of the zone. The total diameter was little affected, but there was a progressive decrease in the width of the intermediate hazy area. Thus, when the inoculum had been so decreased as to give a semi-confluent lawn of corynebacterium, there was little difference in the appearance of the zones produced by $\mathrm{DI}+\mathrm{S}$ and $\mathrm{DI}+\mathrm{H}$ staphylococcal strains.

The size of the initial inoculum of staphylococcus was of little importance. An increase beyond that necessary to give rise to a confluent macrocolony did not result in any increase in the width of zone, and a decrease in inoculum size which resulted in semiconfluent growth, or even discrete colonies, was associated with only a small decrease in zone size. Indeed recognizable zones were formed round well-separated single colonies.

Unsuccessful attempts were made to demonstrate the inhibitory agent in extracts of agar media on which active staphylococci had grown. A number of $\mathrm{DI}+\mathrm{S}$ and $\mathrm{DI}+\mathrm{H}$ strains were grown on semi-solid nutrient agar for $18 \mathrm{hr}$. Fluid was extracted by freezing and thawing and was freed from living staphylococci by heating for $30 \mathrm{~min}$. at $65^{\circ}$ or by passage through sintered glass filters, and tested for activity on lawns of Corynebacterium diphtheriae in punch-holes or Heatley cups. The agent was unsuccessfully sought in the bottom layer of plates prepared by Gratia's filter-paper method. The agar was extracted with broth, with aseptic precautions, and tested for inhibitory action without further treatment. Broth cultures of active staphylococci grown aerobically at $25^{\circ}$ and $37^{\circ}$ for periods up to 6 days were tested for activity after heating at $65^{\circ}$ for $30 \mathrm{~min}$., or after filtration or treatment with chloroform. Undiluted broth did not prevent the growth of as few as four $C$. diphtheriae organisms. However, small numbers of living staphylococci had a pronounced inhibitory effect on the growth of $C$. diphtheriae in liquid media. In one experiment $C$. diphtheriae No. 51 could not be recovered from 1 loopful of blood broth $24 \mathrm{hr}$. after it had been inoculated along with an active staphylococcus in a ratio of $10^{5}: 1$.

\section{Inhibition of other organisms by Staphylococcus aureus}

Other corynebacteria. Dujardin-Beaumetz (1932) and Duliscouët (1935) both found that active staphylococci inhibited the growth of all the corynebacteria they examined. In the earlier stages of the present work several strains of Corynebacterium diphtheriae were tried, and all appeared to be equally susceptible to the action of Staphylococcus aureus Type 71. Later Dr H. G. M. Smith 
(Bradford) sent us a corynebacterium said to be susceptible to inhibition by Type 71 but not by other staphylococci. This Bradford diphtheroid gave sharp zones of inhibition, $2 \mathrm{~mm}$. or more wide, with all $D I+S$ staphylococcus strains and was not inhibited by most $\mathrm{DI}+\mathrm{H}$ strains. It had been isolated from an ear swab and was a short broad bacillus forming flat, whitish, opaque and very friable colonies with lobate edges and a dull granular surface. Diphtheroids resembling this strain culturally are commonly grown from swabs of ear and vaginal discharges. We isolated 9 such strains and found that they all behaved similarly to the Bradford diphtheroid in the inhibition test, though they were not a homogeneous group biochemically.

A collection of $\mathbf{4 0}$ corynebacteria, which included representative strains of Corynebacterium diphtheriae, some atypical diphtheria bacilli, one or more strains each of C. ulcerans, C. ovis, C. pyogenes, C. haemolyticum, C. equi, C. hofmanni, C. xerose, C. fimi and 19 unnamed diphtheroids, was next tested for inhibition by Staphylococcus aureus. The Bradford diphtheroid and 9 strains selected for their cultural resemblance to it were included. Each corynebacterium was tested for inhibition by a number of $S$. aureus strains previously found to be active against $C$. diphtheriae No. 51 by the routine technique. As this took several weeks to carry out, each staphylococcus was tested against C. diphtheriae No. 51 at the beginning and at the end of the series, and only those which gave rise to a zone of inhibition at least $1 \mathrm{~mm}$. wide on both occasions and which showed much the same zone size in the two tests will be considered. Successful tests were carried out with 16 S. aureus DI + Type 71 strains, with 18 others giving sharp zones and with 30 giving hazy zones on C. diphtheriae No. 51. The results are summarized in Table 2.

(i) Five strains of Corynebacterium diphtheriae mitis (including No. 51), one gravis, and three aberrant gravis-like organisms were inhibited by all, or nearly all, of the staphylococcal strains. The characteristic differences between the sharp and hazy type of inhibition zone was apparent in all instances. The growth of none of the corynebacteria in the group was stimulated by any staphylococcus.

(ii) Eleven strains were inhibited by a proportion of staphylococci in each group: nine by all or nearly all. With the exception of Corynebacterium ovis, these strains were diphtheroids which grew either as a thin dry sheet in which the zones were wide and usually surrounded by a zone of enhancement of growth, or as a thick creamy growth in which zones were narrower and rather ill-defined. In none could any distinction between sharp and hazy zones be made out.

(iii) Eleven strains (including the Bradford diphtheroid) were inhibited by all or most Type 71 staphylococcal strains, but by few of the hazy zone producers. All nine of the diphtheroids selected for their colonial resemblance to the Bradford diphtheroid were in this group. All except one of them also showed considerable enhancement of growth around the zone of inhibition. The only other strain falling into this group was one of the three representatives of Corynebacterium xerose.

(iv) Six strains were more often inhibited by hazy-zone-producing staphylococci than by others. The most characteristic members of this group were 
Table 2. Inhibition of other corynebacteria by strains of Staphylococcus aureus active against Corynebacterium diphtheriae strain 51

$$
\begin{aligned}
& \text { S = Strains giving sharp zone on strain } 51 \\
& H=\text { Strains giving hazy zone on strain } 51 \\
& \text { MPHL = Manchester Public Health Laboratory } \\
& \text { NCTC = National Collection of Type Cultures } \\
& \text { BPHL = Bradford Public Health Laboratory }
\end{aligned}
$$

No. of strains of $S$. aureus active

No.

Description

C. diphtheriae mitis

C. diphtheriae mitis

C. diphtheriae mitis

C. diphtheriae mitis

C. diphtheriae mitis

C. diphtheriae gravis

C. diphtheriae aberrant

C. diphtheriae aberrant

C. diphtheriae aberrant

(ii) 10

C. ovis

C. hofmanni

C. xerose

C. hofmanni

C. xerose

C. fimi

C. sp.

C. sp.

C. sp.

C. sp.

C. sp.

(iii) 21

C. sp. 'Bradford'

C. sp. 'Bradford-like'

C. sp. 'Bradford-like'

C. sp. 'Bradford-like'

C. sp. 'Bradford-like'

C. sp. 'Bradford-like'

Origin

MPHL (No. 51)

MPHL

MPHL

MPHL

MPHL

MPHL

MPHL

MPHL

MPHL

NCTC 3450

NCTC 8338

NCTC 7238

NCTC 231

NCTC 8481

NCTC 7547

NCTC 7491

NCTC 764

NCTC 8484

NCTC 934

MPHL

BPHL

MPHL

MPHL

MPHL

MPHL

MPHL

C. sp. 'Bradford-like'

MPHL

C. sp. 'Bradford-like'

MPHL

C. sp. 'Bradford-like'

MPHL

C. sp. 'Bradford-like'

MPHL

C. xerose

NCTC 74,23

(iv) 32

33

C. diphtheriae inter-

NCTC 3987 medius

C. diphtheriae inter-

NCTC 3988

medius

C. ulcerans

MPHL

C. pyogenes

C. equi

NCTC 5224

NCTC 1621

C. sp.

MPHL

(v) 38

C. haemolyticum

NCTC 8452

NCTC 8483

\begin{tabular}{|c|c|c|}
\hline \multicolumn{2}{|c|}{$\mathbf{S}$} & \multirow[t]{2}{*}{$\mathbf{H}$} \\
\hline Type 71 & Other & \\
\hline 16 & 18 & 30 \\
\hline 16 & 18 & $\mathbf{3 0}$ \\
\hline 16 & 9 & 30 \\
\hline 16 & 8 & 30 \\
\hline 16 & 7 & 30 \\
\hline 15 & 8 & 22 \\
\hline 16 & 18 & $\mathbf{3 0}$ \\
\hline 15 & 6 & 12 \\
\hline 16 & 8 & 30 \\
\hline 15 & $\mathbf{5}$ & 9 \\
\hline 13 & 8 & $\mathbf{2 6}$ \\
\hline 16 & 13 & 8 \\
\hline 11 & 18 & 30 \\
\hline 14 & 15 & 19 \\
\hline 16 & 16 & $\mathbf{3 0}$ \\
\hline 16 & 16 & 24 \\
\hline 13 & 12 & $\mathbf{3 0}$ \\
\hline 16 & 17 & 29 \\
\hline 11 & 13 & $\mathbf{3 0}$ \\
\hline 14 & 6 & $2 \gamma$ \\
\hline 16 & 18 & $\mathbf{1}$ \\
\hline 15 & 9 & 3 \\
\hline 14 & 5 & 0 \\
\hline 15 & 6 & 0 \\
\hline 16 & 13 & $\mathbf{0}$ \\
\hline 15 & 8 & $\mathbf{3}$ \\
\hline 15 & 10 & $\mathbf{2}$ \\
\hline 15 & 12 & 4 \\
\hline 16 & 18 & 0 \\
\hline 16 & 15 & $\mathbf{1}$ \\
\hline 16 & 8 & 0 \\
\hline 7 & $\mathbf{3}$ & 28 \\
\hline 2 & 2 & 11 \\
\hline 0 & 0 & 20 \\
\hline $\mathbf{0}$ & 0 & $\mathbf{2 4}$ \\
\hline $\mathbf{0}$ & $\mathbf{3}$ & 28 \\
\hline 4 & 1 & 25 \\
\hline $\mathbf{0}$ & o & $\mathbf{0}$ \\
\hline $\mathbf{0}$ & 0 & 1 \\
\hline 2 & 2 & 0 \\
\hline
\end{tabular}

39
40

C. $\mathrm{sp}$.

C. sp.

MPHL 
Corynebacterium ulcerans, C. pyogenes, $C$. equi and one diphtheroid producing a creamy growth on blood agar. The zones were all rather narrow or ill-defined. The two strains of $C$. diphtheriae intermedius produced wide zones that were so faint as to be almost invisible.

(v) Three strains, including Corynebacterium haemolyticum, were completely or almost completely insusceptible to inhibition.

Staphylococcal strains other than members of Type 71 which produced sharp zones of inhibition of Corynebacterium diphtheriae No. 51 were, on the whole, less active against other corynebacteria than Type 71 strains. It was not possible by inspection of the results to define any sub-patterns of activity that might be useful in classification.

Other Gram-positive organisms. As a first step, 16 strains of Staphylococcus aureus including both $\mathrm{DI}+\mathrm{S}$ and $\mathrm{DI}+\mathrm{H}$ strains, and representatives of all phage groups, were tested for mutual activity by a simultaneous antagonism technique, the overlapping drop method of Rosebury et al. (1954). Also, representative $\mathrm{DI}+\mathrm{S}$ and $\mathrm{DI}+\mathrm{H}$ strains were tested against 46 unselected $S$. aureus strains, and 15 coagulase-negative staphylococci. No evidence of inhibition was obtained, suggesting that powerful agents active on Grampositive cocci were not very common in $S$. aureus. However, when the more sensitive deferred antagonism technique of Abbott \& Shannon (1958) was used, evidence of inhibition of some staphylococci by others was obtained. All the active strains were $\mathrm{DI}+\mathrm{S}$ and they inhibited all the $\mathrm{DI}-$ and $\mathrm{DI}+\mathrm{H}$ strains against which they were tested. The results of a typical experiment are shown in Table 3. Some of the reactions were quite weak and in most cases some resistant colonies were visible in the areas of inhibition. Further experiments showed that several DI+ ' 71 -like' members of phage Group II had a similar effect on other staphylococci. It seemed possible, therefore, that the same agent might be responsible for both the sharp-zone inhibition of Corynebacterium diphtheriae and for the inhibition of other $S$. aureus strains. Some support for this was obtained from an experiment in which a DI +S Type 7I strain and a DI - variant derived from it were tested for mutal inhibition: the DI - variant was inhibited by the DI + parent strain, but itself had no inhibitory activity.

Experiments were also carried out, using Abbott \& Shannon's technique, in which 14 strains of Staphylococcus aureus (including $4 \mathrm{DI}+\mathrm{S}$ and $5 \mathrm{DI}+\mathrm{H}$ strains) were tested for activity on 7 coagulase-negative staphylococci, two pneumococci and 12 haemolytic streptococci (4 each of groups A, C and G). Each $S$. aureus strain gave evidence of inhibition of one or more organism, but the pattern of activity was extremely complex, and the DI + strains did not appear to behave in a uniform fashion.

\section{Loss of power to inhibit corynebacteria}

Early in the investigation it was observed that Staphylococcus aureus Type 71 strains lost their ability to inhibit corynebacteria on storage in the laboratory. This took place regularly between the 3rd and 12th month in agar stabs kept at room temperature. When subcultures were tested at intervals, the zone of inhibition was seen to become progressively narrower, and by the 5th-6th 


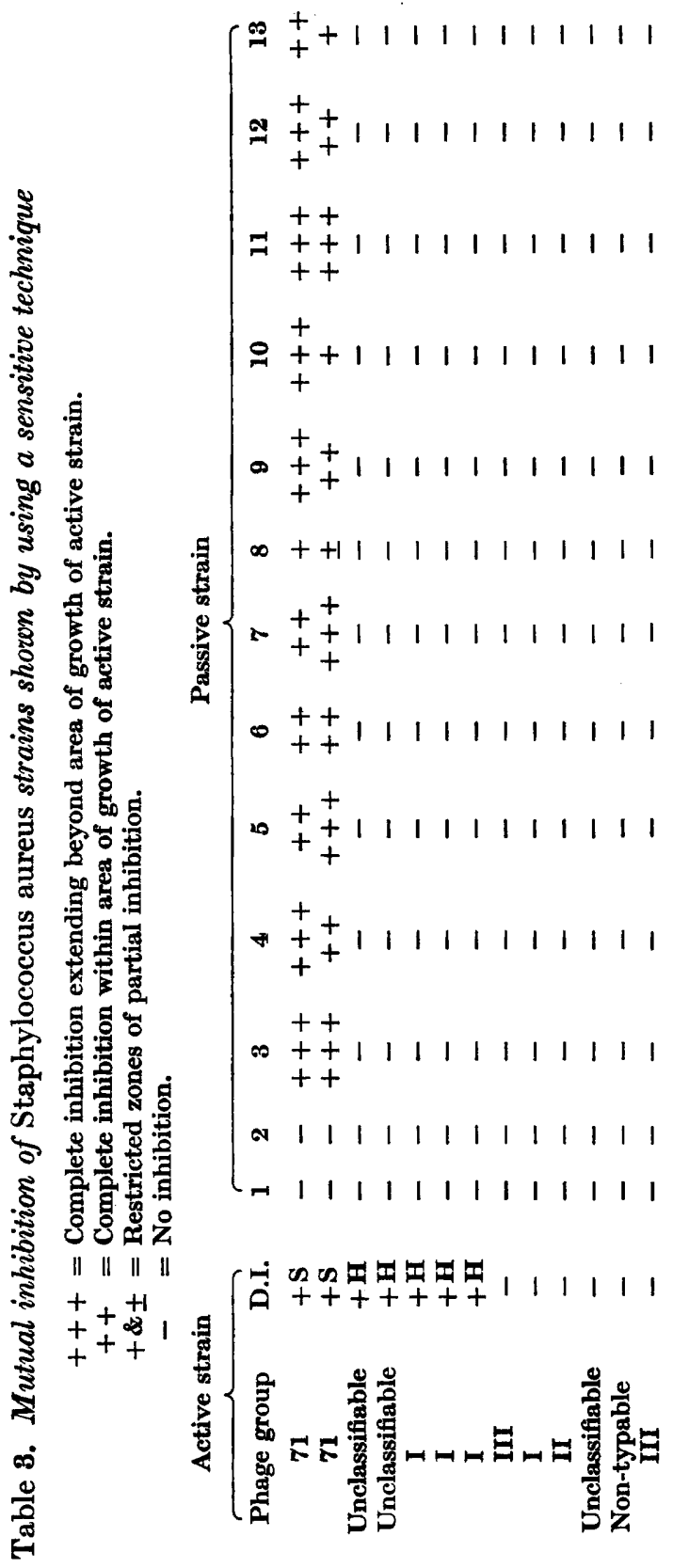


month was often reduced to a fine crevasse between the growth of the staphylococcus and the corynebacterium. Loss of inhibitory power was not associated regularly with any other change in cultural characteristics : most strains retained their original ability to form pigment, coagulase, haemolysin and hyaluronidase. Most were penicillin resistant and egg-yolk negative on first isolation and these properties were unchanged. Table 4 shows the results of 885 inhibition tests on Type 71 staphylococcal strains at various times after isolation; strains giving zones $1 \mathrm{~mm}$. or more in radius in the routine test were considered to be still $\mathrm{DI}+$. The results include those of the initial test on 534 strains (most, but not all, being done within 3 months of isolation) together with repetitions carried out on unselected batches of cultures. There was a small decrease in the proportion of positive results between the 3rd and 6th months and a much greater decrease subsequently; only 1 of 70 tests carried out after 12 months was positive. Staphylococci other than members of Type 71 which produced sharp zones of inhibition showed a similar loss of activity on storage, and nearly all were DI - after 1 year.

Table 4. Results of inhibition tests on cultures of Staphylococcus aureus Type 71 stored in the laboratory for different periods of time

\begin{tabular}{lrrrrr} 
& \multicolumn{5}{c}{ Months of storage } \\
\cline { 2 - 6 } & $<3$ & $8-6$ & $6-9$ & $9-12$ & $>12$ \\
Number tested & 481 & 211 & 76 & 47 & 70 \\
Number inhibitory & 450 & 180 & 44 & 2 & 1 \\
$\%$ inhibitory & 94 & 85 & 58 & 4 & 1
\end{tabular}

The ability to produce hazy zones of inhibition appeared to be unaffected by prolonged storage in the laboratory. Twenty-nine staphylococcal strains re-tested 12-21 months after isolation were active and gave zones as large as those seen when they were first tested. A culture of Staphylococcus aureus (NCTC 6507), which was described by A. Fleming as an inhibitor of Corynebacterium diphtheriae when deposited in the National Collection of Type Cultures in 1943 produced a good zone of hazy inhibition in 1955.

The nature of this loss of activity was investigated by a method which makes use of the fact that single colonies of a DI + staphylococcus deposited on a lawn of Corynebacterium diphtheriae of somewhat decreased thickness give rise to recognizable zones of inhibition. Loopfuls of a broth culture of a staphylococcus so diluted as to yield 20-30 colonies each were deposited on a fresh lawn of $C$. diphtheriae made by flooding a blood agar plate with a broth emulsion about $1 / 10$ of the strength of that used in the routine inhibition test. When Type 71 staphylococcal strains, which gave somewhat narrowed zones of inhibition in the routine test were treated in this manner, two sorts of colonies were seen: larger, well-developed colonies surrounded by clear-cut zones of inhibition about $0.5 \mathrm{~mm}$. in radius, and rather smaller colonies with no zone around them (Pl. 1, fig. 4). On subculture to blood agar the two sorts of colony were indistinguishable; the difference on the original plates was undoubtedly due to crowding of the non-inhibiting colonies by the 
corynebacteria. When the routine test was carried out on these subcultures, those from inhibiting colonies gave sharp zones of full width and those from non-inhibiting colonies were inactive. Repetition of the tests over a period of some weeks gave identical results, but when the selected variants were stored for some months the inhibition zones of the DI + strains again became narrower and the cultures were again found to consist of mixtures of DI + and DI variants. Repeated examination of individual colonies from artificially selected DI - variants did not reveal the presence of any DI + forms.

\section{Changes in susceptibility to typing phages associated with loss of inhibitory activity}

When strains of Staphylococcus aureus Type 71, which on first isolation had been lysed only by phage 71 at RTD, were re-typed some months later it was found that many of them had also become susceptible to phage 55 at RTD. This appeared to occur after about the same period of storage as the loss of ability to inhibit Corynebacterium diphtheriae. The association between the two changes was therefore investigated in 79 originally DI + members of $S$. aureus Type 71 which had been isolated 5-30 months previously (Table 5). Sixty-two

Table 5. Acquisition of sensitivity to phage 55 by Staphylococcus aureus Type 71 strains, and its relation to loss of ability to inhibit Corynebacterium diphtheriae

$\mathrm{DI}+=$ zone of inhibition $>1 \mathrm{~mm}$. radius.

Lysis by phage $55=++=>49$ plaques; $+=20-49$ plaques; $\pm=1-19$ plaques.

Lysis by phage 55

\begin{tabular}{|c|c|c|c|c|c|c|c|}
\hline \multicolumn{4}{|c|}{ DI - } & \multicolumn{4}{|c|}{$\mathrm{DI}+$} \\
\hline++ & + & \pm & - & ++ & + & \pm & - \\
\hline 39 & 3 & 2 & 1 & 0 & 0 & 0 & 1 \\
\hline 18 & 0 & 0 & 2 & $1 *$ & 0 & 0 & 12 \\
\hline
\end{tabular}

of the 65 strains which had become DI - were now lysed by phage 55; 57 of them reacted strongly. All but 1 of the 14 strains still DI + were insusceptible to phage 55. The one exception was, in fact, an example of a more far-reaching change in phage pattern, as it was now also lysed by phages $\mathbf{3 A}, \mathbf{3 B}$ and $\mathrm{3C}$. There was, therefore, a general tendency for the two changes to be associated, but exceptions occurred. However, it was also observed that 3 other members of Type 71 that were DI - on first isolation, also became fully susceptible to phage 55 within 1 year.

A small number of staphylococcal strains which failed to react with phage 71 at RTD but were lysed by phage 71 (and usually by other Group II phages) at $1000 \times$ RTD were included in Type 71 by Parker, Tomlinson \& Williams (1955). Twelve of these so-called 'weak 71' strains, which were DI+ on first isolation, were re-tested after periods exceeding 1 year, by which time they had all become DI - . Eight now reacted with phage 71 at RTD, 1 with phage 55 and 1 with'both 55 and 71 : two were unchanged. Ten '71-like' strains 
from impetigo lesions, originally completely non-typable at RTD and at $1000 \times$ RTD, but all DI + S, were also re-examined over one year after isolation. All were now DI - : two were lysed by both phages 55 and 71, 4 by 71, 3 by 55 and one by Group I phages, all at RTD.

It appeared, therefore, that no one phage pattern was exclusively associated with the presence or absence of inhibitory activity. Whatever the phage pattern of the original $\mathrm{DI}+\mathrm{S}$ form, loss of inhibitory power was associated with a gain of susceptibility to Group II phages, and susceptibility to phage 71 usually but not always 'preceded' susceptibility to phage 55 in the course of dissociation.

Some of the discrepancies in the results shown in Table 5 might have been due to the fact that some of the staphylococcal cultures were in fact mixtures of $\mathrm{DI}+$ and DI - forms. Therefore, a number of freshly-selected DI + and DI variants were obtained from cultures which gave narrow zones in the routine inhibition test, using the technique described in the previous section, and were re-typed. Table 6 shows the susceptibility to phage 55 of 118 such variants from 12 cultures. The general association of susceptibility to lysis by phage 55 with absence of inhibitory power was again seen: with most strains the difference was quite clear cut, but discrepancies occurred in a minority of strains.

Table 6. Lysis by phage 55 of artificially-selected $\mathrm{DI}+$ and $\mathrm{DI}-$ variants from twelve Staphylococcus aureus Type 71 cultures

Number of variants lysed to the degree shown.

\begin{tabular}{|c|c|c|c|c|c|c|c|c|}
\hline \multirow[b]{4}{*}{$\begin{array}{l}\text { Staphylococcal } \\
\text { strain no. }\end{array}$} & \multicolumn{8}{|c|}{ Degree of lysis by phage 55} \\
\hline & \multicolumn{4}{|c|}{ DI - variants } & \multicolumn{4}{|c|}{ DI + variants } \\
\hline & $+t$ & + & \pm & - & $+t$ & + & \pm & - \\
\hline & & & & & & & & \\
\hline 1 & $\mathbf{5}$ & . & . & . & . & . & . & $\mathbf{5}$ \\
\hline $\mathbf{2}$ & $\mathbf{5}$ & . & . & . & . & . & . & 5 \\
\hline $\mathbf{3}$ & $\mathbf{5}$ & . & . & . & . & . & . & $\mathbf{5}$ \\
\hline 4 & 5 & . & . & . & . & . & . & $\mathbf{5}$ \\
\hline $\mathbf{5}$ & $\mathbf{5}$ & . & . & . & . & - & - & $\mathbf{5}$ \\
\hline 6 & $\mathbf{5}$ & . & . & . & . & . & - & 5 \\
\hline 7 & 5 & . & . & . & . & . & . & $\mathbf{8}$ \\
\hline 8 & 2 & - & . & . & . & . & . & $\mathbf{1}$ \\
\hline $\mathbf{9}$ & 4 & . & 1 & . & . & . & . & 5 \\
\hline 10 & 10 & . & - & . & $1^{*}$ & . & . & 9 \\
\hline 11 & 2 & . & . & . & 1 & 1 & $\mathbf{2}$ & 1 \\
\hline \multirow[t]{2}{*}{12} & 5 & . & . & . & $\mathbf{2}$ & 2 & . & 1 \\
\hline & 58 & 0 & 1 & 0 & 4 & $\mathbf{3}$ & $\mathbf{2}$ & 50 \\
\hline
\end{tabular}

Rountree (1956) attributed the resistance of Staphylococcus aureus strains to certain typing phages to the presence of symbiotic phage; this suggested that the step-wise gain in susceptibility to certain Group II phages which accompanies loss of inhibitory power might also be due to the loss of a phage. An attempt was therefore made to obtain a phage from the DI + form with which to lysogenize the DI-form. The two were grown together under a variety of conditions, but no such phage could be detected. 
Attempts to induce lysis in mixtures by ultraviolet irradiation were also unsuccessful. Variants that were DI - and susceptible to phage 55 were successfully lysogenized with. this phage and so rendered resistant to its action; but they remained DI - .

Among the artificially-selected variants shown in Table 6 was one with a grossly aberrant phage pattern (strong lysis by phages $3 \mathrm{~A}, 3 \mathrm{~B}, 3 \mathrm{C}, 55$ and 71) which had retained its inhibitory activity. This appeared to be of some significance, since a whole culture which underwent the same change (71 to $3 \mathrm{~A} / 3 \mathrm{~B} / \mathrm{3C} / 55 / 71$ ) while remaining $\mathrm{DI}+\mathrm{S}$ had been seen previously (Table 5 ), and also since a DI $+S$ strain with the pattern $3 \mathrm{~A} / 3 \mathrm{~B} / 3 \mathrm{C} / 55 / 71$ had once been isolated from a case of impetigo. The artificially-selected variant $3 \mathrm{~A} / \mathbf{3} \mathrm{B} / \mathbf{3 C} /$ $55 / 71: D I+S$ had been found in a culture together with both the $71: D I+S$ and the 55/71:DI - forms, and when either of these forms was grown in broth the supernatant fluid contained a phage lytic for it. Phages derived from both forms were identical in their lytic spectrum on the standard propagating strains (strong lysis of PS $3 \mathrm{C}$ and weak lysis of PS 3A). Strong preparations of both phages were made by growth on $3 \mathrm{~A} / 3 \mathrm{~B} / \mathbf{3 C} / 55 / 71$, and were spotted on lawns of the same strain. Subcultures from the resistant growth were made, and from them a strain lysed only by phage 71 , but also $\mathrm{DI}+\mathrm{S}$, was obtained. The $3 \mathrm{~A} / 3 \mathrm{3B} / 3 \mathrm{C} / 55 / 71$ variant did, therefore, appear to have arisen from a loss of symbiotic phage; but the change was not accompanied by any alteration in the ability to inhibit corynebacteria.

Naturally-occurring staphylococcal cultures with the phage-pattern 55/71

The regular appearance of staphylococcal variants with the phage pattern $55 / 71$ in laboratory cultures originally lysed only by phage 71 stimulated interest in naturally-occurring strains with the same pattern. The 55/71 variants had the same cultural characters as their parent Type 71 strains except for the negative DI reaction, i.e. they were egg-yolk negative and usually penicillin resistant. Nearly all naturally-occurring members of phage Group II, other than Type 71, are egg-yolk positive and usually penicillin sensitive. Further, Type 71 strains very seldom give rise to deep suppuration, while other members of phage Group II frequently do. In the series of 2032 cultures summarized in Table 1 there were 286 members of phage Group II other than Type 71, and 39 of them reacted only with phages 55 and 71 . The origin and cultural characters of these $55 / 71$ strains are shown in Table 7. Fifteen of them, including 6 from deep infections, resembled 'the rest' of phage Group II in being egg-yolk positive and DI - : all were penicillin sensitive. The other 24, all from impetigo or other vesicular skin lesions, were egg-yolk negative, all but one were penicillin resistant, but less than half were DI + . The DImembers of this latter group were, in fact, indistinguishable from DI variants arising from Type 71 cultures in vitro. Such strains were encountered on several occasions in outbreaks of pemphigus neonatorum in which lesions of closely-related cases had yielded ordinary Type 71 strains. This had led to some difficulty in interpreting the typing results, but we had concluded that the strains were epidemiologically connected. An opportunity to investigate 
this situation further arose in connexion with a severe case of pemphigus neonatorum in a child born at home. Four cultures were obtained, one each from the infant's skin and nose, and one from each nostril of the midwife. All were typed and tested for inhibition of Corynebacterium diphtheriae within 1 week of isolation. The baby's skin strain was 55/71:DI - and his nose strain $71: D I+S$. Of the two strains from the midwife, one was 55/71:DI and the other, although thought to have been obtained from a single-colony 'pick' was a mixture of 55/71:DI - and $71: \mathrm{DI}+\mathrm{S}$. It must be assumed, therefore, that the population in both the patient. and the nurse was a mixed one.

\section{Table 7. Origin and cultural characteristics of 39 naturally-occurring staphylococcal strains with phage-pattern 55/71}

\begin{tabular}{|c|c|c|c|c|}
\hline \multirow[b]{3}{*}{ Origin of strains } & \multicolumn{4}{|c|}{ Egg-yolk reaction } \\
\hline & \multicolumn{2}{|c|}{+} & \multicolumn{2}{|c|}{-} \\
\hline & DI + & DI - & $\mathrm{DI}+$ & DI - \\
\hline Impetigo & $\mathbf{0}$ & 5 & 6 & $\mathbf{2}$ \\
\hline Pemphigus neonatorum & $\mathbf{0}$ & $\mathbf{0}$ & 4 & 12 \\
\hline Deep suppuration & $\mathbf{0}$ & 6 & $\mathbf{0}$ & 0 \\
\hline Normal nose & 0 & 4 & $\mathbf{0}$ & 0 \\
\hline
\end{tabular}

\section{DISCUSSION}

The observations recorded in this paper were made incidentally during an enquiry into the epidemiology of superficial skin infections. Many earlier workers had studied the inhibition of corynebacteria and other Grampositive organisms by strains of Staphylococcus aureus, but their interest had been concentrated mainly on the mechanism of the inhibition, and on the part it might play in clearing the upper respiratory tract of Corynebacterium diphtheriae. The ability to inhibit appeared to be distributed in a haphazard fashion among strains of staphylococci and there was little indication that it might be a characteristic of a certain sort of staphylococcus. It was clear, however, that different staphylococci produced a variety of inhibitory agents, and that the number and type revealed would depend on the precise method used to detect them. It was fortunate that the simple method adopted by Tomlinson in his early work on impetigo should have been so well adapted for the detection of the agent produced by $S$. aureus Type 71. Members of phage Type 71 and a small number of related strains, all of which were associated with superficial vesicular skin lesions, produced a characteristic, narrow, sharply-defined zone. A further group of staphylococci produced an easily distinguishable zone with a hazy periphery: these strains had little else in common, except their lack of association with staphylococcal infections in man.

In recent years a number of bacterial species and serotypes have been split up into epidemiologically valid subdivisions by determining the range of activity of the inhibitory substances produced by individual strains. Fredericq, Betz-Bareau \& Nicolle (1956) and Shannon (1957) produced typing systems for certain O-groups of Escherichia coli, Abbott \& Shannon (1958) were able 
to divide Shigella sonnei into 7 colicine types and Thibaut \& Fredericq (1956) further subdivided a group of Corynebacterium diphtheriae strains insusceptible to typing phages. In general, however, the members of the colicine or bacteriocine types so defined do not appear to differ from each other except in their ability to produce inhibitory agents, though Thibaut \& Fredericq (1956) did observe that strains of $\boldsymbol{C}$. diphtheriae gravis not producing agents active against certain indicator organisms were invariably fermenters of glycogen. So far, however, there has been no instance, other than that recorded here, of the production of a particular inhibitory agent only by strains with the ability to cause a particular sort of lesion.

Both the sharp and the hazy zones of inhibition of Corynebacterium diphtheriae appear to be due to agents produced by staphylococci during growth, which diffuse slowly through agar but not through cellophan, and which kill C. diphtheriae. Further investigation of the properties of these agents was hampered by our failure to obtain cell-free preparations of them. Others have found little difficulty in separating and concentrating the colicines (see Goebel, Barry, Jesaitis \& Miller, 1955), the pyocines (Hamon, 1956) and the anti-staphylococcal agents produced by staphylococci (Halbert $e t$ al. 1953). However, Thibaut \& Fredericq (1956) found that the agents produced by C. diphtheriae which inhibited other corynebacteria also diffused slowly in agar and could not be demonstrated in broth cultures.

There are several important differences between the agents responsible for sharp and for hazy zones of inhibition of Corynebacterium diphtheriae. Their range of activity on other corynebacteria is different: all or most of the staphylococci which inhibit the strain of $C$. diphtheriae mitis used in our routine test also inhibit a number of other corynebacteria, including other mitis and gravis strains and a number of diphtheroids, but a group of strains with recognizable colonial characters was found to be susceptible to inhibition by sharp zone forming but not by hazy zone forming staphylococci. Also, staphylococci which give sharp zones of inhibition of $C$. diphtheriae have a weak but definite inhibitory action on staphylococci not able to produce sharp zones, and there is some evidence that both activities are due to the same substance. On the other hand, strains forming hazy zones on $C$. diphtheriae had no action on other staphylococci.

Although the ability to produce hazy zones of inhibition persists indefinitely in culture, the ability to produce sharp zones is regularly lost after several months. This change takes place in one step and is associated with a broadening of susceptibility to lysis by typing phages. Staphylococcal strains at first lysed by phage 71 at RTD become susceptible also to phage 55, strains lysed by Group II phages only at $1000 \times$ RTD become more susceptible to phage 71, or less often to phage 55, and strains previously untypable at $1000 \times$ RTD become susceptible in many instances to phage 71, or to phage 55. This provides further evidence that certain 71-like strains are closely related to Staphylococcus aureus Type 71.

The authors are indebted to Miss Jean Perry of the Department of Medical Illustration, Crumpsall Hospital, Manchester, for the photographs shown in the Plate. 


\section{REFERENCES}

Aвbott, J. D. \& Shannon, R. (1958). A method for typing Shigella sonnei using colicine production as a marker. J. clin. Path. 11, 71 .

Anderson, E. S. \& Williams, R. E. O. (1956). Bacteriophage typing of enteric pathogens and staphylococci and its use in epidemiology. J. clin. Path. 9, 94.

Dujardin-Beaumetz, E. (1932). Action antibiotique d'une variété de staphylocoque a l'égard des bacilles Gram-positifs et acido-résistants. C.R. Soc. Biol., Paris, 110, 1210.

Dulrscouër, R. (1935). Action probiotique et antibiotique des staphylocoques chez les porteurs des germes diphtériques. C.R. Soc. Biol., Paris, 118, 1277.

DulIscouËr, R. (1939). Recherches experimentales sur une substance bactériolytique dérivée des germes antagonistes. Les applications dans la thérapeutique de la diphtérie. Arch. Méd. Pharm. Nav. 129, 410.

DuliscouËt, R. (1945). Un antagoniste microbien nouveau. La staphyline antidiphtérique. $\operatorname{Pr}$. méd. 53, 653.

FredericQ, P. (1946). Sur la sensibilité et l'activité antibiotique des staphylocoques. C.R. Soc. Biol., Paris, 140, 1167.

FredericQ, P. (1948). Actions antibiotiques réciproques chez les Enterobacteriaceae. Rev. Belge Path. 19, Supp. IV.

FredericQ, P., Betz-Bareau, M. \& Nicolle, P. (1956). Typage des souches d'Escherichia coli de gastro-entérite infantile par recherche de leurs propriétés colicinogènes. C.R. Soc. Biol., Paris, 150, 2039.

Goebel, W. F., Barry, G. T., Jesaitis, M. A. \& Miluler, E. M. (1955). Colicine K. Nature, Lond. 176, 700.

Gratia, A. (1925). Sur un remarquable exemple d'antagonisme entre deux souches de colibacille. C.R. Soc. Biol., Paris, 93, 1040.

Gratia, A. (1946). Techniques sélectives pour la recherche systématique des germes antibiotiques. C.R. Soc. Biol. Paris, 140, 1053.

Halbert, S. P., Swick, L. \& Sonv, C. (1953). Characteristics of antibiotic-producing strains of the ocular bacterial flora. J. Immunol. 70, 400.

Hamon, Y. (1956). Contribution a l'étude des pyocines. Ann. Inst. Pasteur, 91, 82.

Jennings, M. A. \& Sharp, A. E. (1947). Antibacterial activity of the staphylococcus. Nature, Lond. 159, 133.

Parker, M. T. (1958). Some cultural characteristics of Staphylococcus aureus strains from superficial skin infections. J. Hyg., Camb. 56, 238.

Parker, M. T., Tomlinson, A. J. H. \& Wrlliams, R. E. O. (1955). Impetigo contagiosa. The association of certain types of Staphylococcus aureus and of Streptococcus pyogenes with superficial skin infections. J. Hyg., Camb. 53, 458.

Rosebury, T., Gale, D. \& TAYlor, D. F. (1954). An approach to the study of interactive phenomena among microorganisms indigenous to man. J. Bact. 67, 135.

Rountree, P. M. (1956). Variations in a related series of staphylococcal bacteriophages. J. gen. Microbiol. 15, 266.

Shannon, R. (1957). Colicine production as a method for typing Bact. coli 055 B5. J. med. Lab. Technol. 14, 199.

Terrasse, G. \& SoHIer, R. (1954). Recherches sur l'activité antibiotique et 'probiotique' de diverses Corynébactéries non pathogènes a l'égard de Corynebacterium diphtheriae. Ann. Inst. Pasteur, 87, 727.

Thibaut, J. (1949). Activité antibiotique des Corynébactéries. C.R. Soc. Biol., Paris, 143, 1150.

Thibaut, J. \& FredericQ, P. (1956). Actions antibiotiques réciproques chez Corynebacterium diphtheriae. C.R. Soc. Biol., Paris, 150, 1512. 


\section{EXPLANATION OF PLATE}

Fig. 1. Inhibition of Corynebacterium diphtheriae by Staphylococcus aureus. Left: sharp zone; Right: hazy zone; $\times 1.5$ Transmitted light.

Fig. 2. Sharp zone of inhibition. $\times 2$. Reflected light.

Fig. 3. Hazy zone of inhibition. $\times 2$. Reflected light.

Fig. 4. Single colonies of $S$. aureus from a stored culture on a lawn of $C$. diphtheriae. Inhibition zones around some colonies but not around others. $\times 4$. Reflected light.

(Received 6 May 1959) 


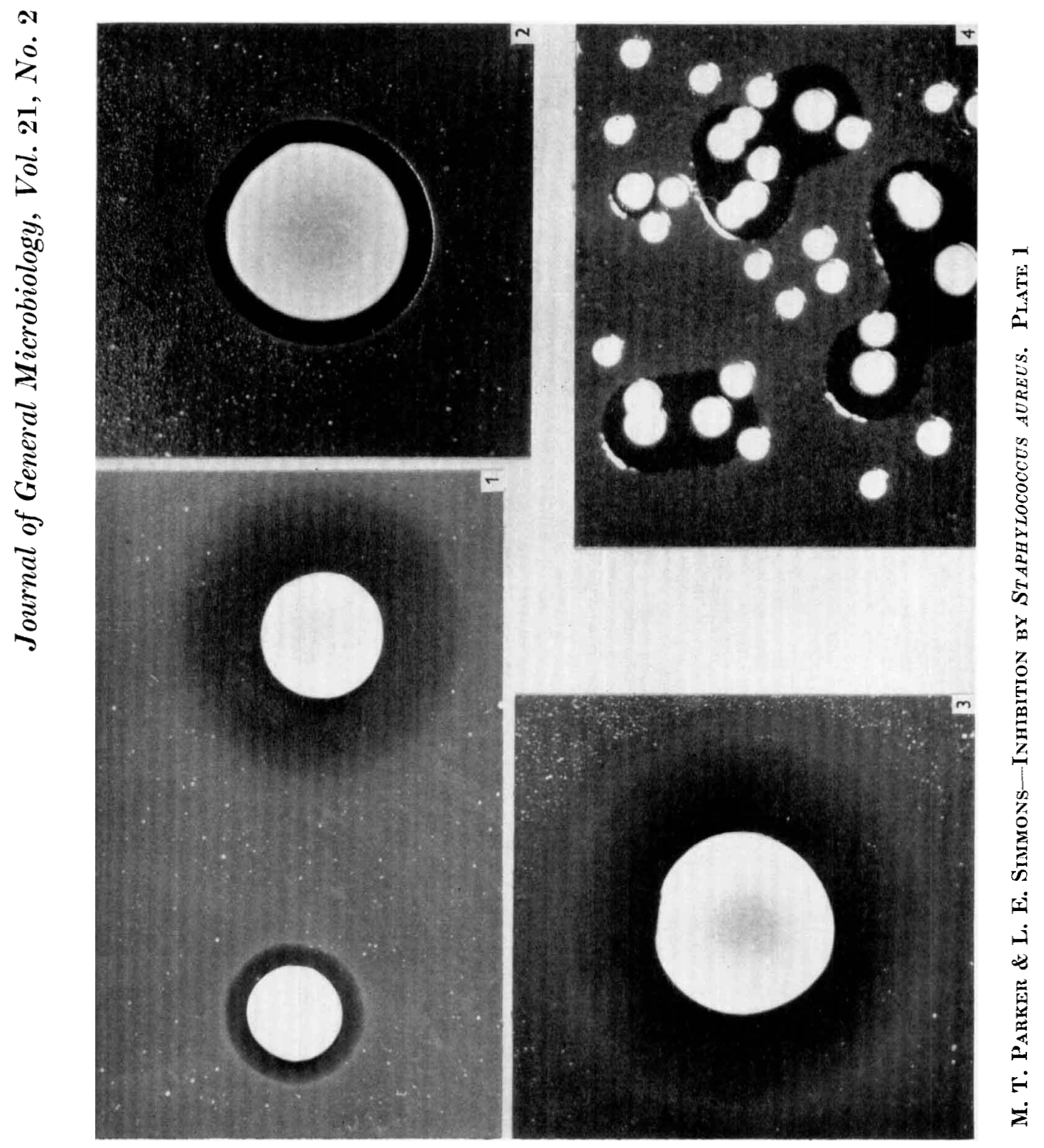

(Facing $p$. 476) 